\title{
Polynomial Lie algebra methods in solving the second-harmonic generation model: some exact and approximate calculations *
}

\author{
V.P. Karassiov ${ }^{a, b}$ A.A. Gusev ${ }^{b}$ S.I. Vinitsky ${ }^{b}$ \\ ${ }^{a}$ P.N. Lebedev Physical Institute, Leninsky Pr. 53, Moscow, 117924 Russia, email: \\ vkaras@sci.lebedev.ru (contact address) \\ b JINR, Dubna, Moscow region, Russia, \\ email: vinitsky@thsun1.jinr.ru
}

\begin{abstract}
We compare exact and $S U(2)$-cluster approximate calculation schemes to determine dynamics of the second-harmonic generation model using its reformulation in terms of a polynomial Lie algebra $s u_{p d}(2)$ and related spectral representations of the model evolution operator realized in algorithmic forms. It enabled us to implement computer experiments exhibiting a satisfactory accuracy of the cluster approximations in a large range of characteristic model parameters.
\end{abstract}

Key words: second-harmonic generation model, polynomial Lie algebra methods PACS: 42.50, 42.65, 02.20, 03.65

\section{Introduction}

During the last half-century a great attention is being paid to studies of different quantum models of nonlinear optics since they enable to reveal new physical effects and phenomena (see, e.g., [1-13] and references therein). In view of the Hamiltonian nonlinearity these models are mainly analyzed with the help of the numerical calculations $[9,19,20]$ or some linearization procedures $[4,7,8]$ which are not adapted to reveal many peculiarities of model dynamics

\footnotetext{
* A financial support of the work from the Russian Foundation for Basic Research under grant No.00-02-81023 Bel 2000_a is acknowledged.
} 
[2,5,9,11-13]. However, recently a new universal Lie-algebraic approach, essentially improving both analytical and numerical solutions of physical problems, has been suggested in [14] and developed in $[6,11,12,15]$ for the class of nonlinear quantum models whose Hamiltonians $H$ have invariance groups $G_{i}:\left[G_{i}, H\right]=0$. This approach is based on reformulations of models under study in terms of dual algebraic pairs $\left(G_{i}, g^{D}\right)[12,14]$ where dynamic symmetry algebras $g^{D}$ are polynomial Lie algebras (PLA) $g_{p d}: g^{D}=g_{p d}$ completely describing model dynamics. Specifically, for a wide class of quantum-optical models with $g^{D}=g_{p d}=s u_{p d}(2)$ both exact and approximate methods were developed in $[6,11,12,14,15]$ to obtain adequate (for physical applications) forms of the model evolution operators $U_{H}(t)$. Exact methods include appropriate Lie-algebraic path-integral schemes [12] and $s u_{p d}(2)$-techniques $[6,14,15]$ based on solving difference and difference-differential equations which are fairly complicated for practical calculations because they yield only algorithms rather than closed analytical expressions for solving physical problems. Therefore, for this aim two analytical approximations of $U_{H}(t)$ were found in [11]. They enable to examine model dynamics at cluster quasiclassical levels described by "large" values of certain characteristic parameters [11,12]. However, up to now systematic examinations of their accuracy and efficiency were absent.

In the present work we cancel in part this lacuna for the simplest and most widely examined (see, e.g. [1,3,4,6-8,16-20] and references therein) example of such models describing process of second-harmonic generation which is given by the Hamiltonian

$$
H=\hbar\left[\omega_{0} a_{0}^{+} a_{0}+\omega_{1} a_{1}^{+} a_{1}+g a_{1}^{+2} a_{0}+g a_{1}^{2} a_{0}^{+}\right]
$$

and by the Fock space $L_{F}(2)=\operatorname{Span}\left\{\left|\left\{n_{1}, n_{0}\right\}\right\rangle \propto\left(a_{1}^{+}\right)^{n_{1}}\left(a_{0}^{+}\right)^{n_{0}}|0\rangle\right\}$ as the model Hilbert space $L(H)$; here $a_{i}, a_{i}^{+}$are operators of field modes with frequencies $\omega_{i}, g$ are coupling constants. Classical solutions of the model (1) were first found (in terms of elliptical functions) in [1], and later its different quantum features were examined by many investigators (see, e.g., [3,4,6-8,11-20] and references therein). Specifically, in $[11,17,19]$ some peculiarities, like the collapse-revival phenomena in the Janes-Cummings model [2], were revealed for its model dynamics at the quantum and quasiclassical levels.

The work is organized as follows. After some preliminaries (Section 2) we give exact and approximate calculation schemes and routines to determine "diagonal" (spectral) representations of the model evolution operator by means of the $s u_{p d}(2)$-techniques $[14,15]$ (Section 3). In Section 4 we implement computer experiments to exhibit their efficiency and to investigate the accuracy of appropriate quasiclassical approximations obtained in [11]. In Conclusion we briefly discuss some of ways to improve the results obtained. 


\section{The $s u_{p d}(2)$ formulation of the model and a related "diagonal" representation of its dynamics}

As was shown in $[12,14]$, the invariance of the model Hamiltonian (1) with respect to the group $G_{i}=C_{2} \times \exp \left(i \lambda R_{1}\right), C_{2}=\left\{\exp \left(i r N_{1}\right): r=0,1\right\}$ enables to re-formulate the model under study in terms of two sets of collective operators: integrals of motion $R_{1}=\left(N_{1}+2 N_{0}\right) / 3, K=N_{1}-2\left[N_{1} / 2\right]\left(N_{i}=\right.$ $a_{i}^{+} a_{i},[A]$ is the entire part of $A$ ) and $G_{i}$-invariant dynamic variables $V_{\alpha=0, \pm}$ :

$$
V_{+}=a_{1}^{+2} a_{0}, \quad V_{-}=a_{0}^{+} a_{1}^{2}, \quad V_{0}=\left(N_{1}-N_{0}\right) / 3
$$

They obey the commutation relations

$$
\begin{aligned}
& {\left[V_{0}, V_{ \pm}\right]= \pm V_{ \pm}, \quad\left[V_{\alpha}, R_{1}\right]=0=\left[V_{\alpha}, K\right]} \\
& {\left[V_{-}, V_{+}\right]=\phi\left(V_{0} ; R_{1}\right) \equiv \psi\left(V_{0}+1 ; R_{1}\right)-\psi\left(V_{0} ; R_{1}\right),} \\
& \psi\left(V_{0} ; R_{1}\right)=\left(R_{1}+2 V_{0}\right)\left(R_{1}+2 V_{0}-1\right)\left(R_{1}+1-V_{0}\right),
\end{aligned}
$$

that identifies $V_{\alpha}$ as generators of PLA $s u_{p d}(2)$ having the Casimir operator

$$
\begin{aligned}
& \Psi\left(R_{0}=\left(K-R_{1}\right) / 2\right) \equiv \psi\left(V_{0} ; R_{1}\right)-V_{+} V_{-}=\psi\left(V_{0}+1 ; R_{1}\right)-V_{-} V_{+}, \\
& {\left[V_{\alpha}, \Psi\left(R_{0}\right)\right]=0}
\end{aligned}
$$

acting on $L(H)$ complementarily to $G_{i}$ (in view of the relationship $K=2 R_{0}+$ $R_{1}$ ) and, hence, forming dynamic symmetry algebra $g^{D}$ in the dual algebraic pair $\left(G_{i}, g^{D}\right)[12,14]$.

In terms of these collective operators the Hamiltonian (1) is expressed in the form $[14]$

$$
\begin{aligned}
& H=\hbar\left[\Delta V_{0}+g V_{+}+g^{*} V_{-}+C\left(R_{1}\right)\right], \\
& C=\left(\omega_{1}+\omega_{0}\right) R_{1}, \quad \Delta=2 \omega_{1}-\omega_{0}, \quad\left[V_{\alpha}, C\right]=0,
\end{aligned}
$$

and the Hilbert space $L(H)$ is decomposed into the infinite direct sum

$$
\begin{aligned}
& L(H)=\sum_{k=0,1} \sum_{s=0}^{\infty} L(k, s), \\
& L(k, s)=\operatorname{Span}\left\{|k, s ; f\rangle: R_{i=0,1}|k, s ; f\rangle=l_{i}|k, s ; f\rangle\right\},
\end{aligned}
$$

of $G_{i} \otimes s u_{p d}(2)$-irreducible $s+1$ - dimensional subspaces $L(k, s)$ specified by eigenvalues $l_{i}$ of the $G_{i} \otimes s u_{p d}(2)$ invariant operators $R_{i}$. Herewith $l_{i}$ are expressed through the numbers $s, k: l_{0}=(k-s) / 3, l_{1}=(k+2 s) / 3$ determining, respectively, a maximal population of the fundamental (pump) mode and 
a minimal population of the harmonic within a fixed "optical atom" $L(k, s)$ as it follows from the structure of the new (collective) basis in $L(H)$ [11]:

$$
\begin{aligned}
& |k, s ; f\rangle=N(f ; k, s) V_{+}^{f}|k, s\rangle=\left|\left\{n_{1}=k+2 f, n_{0}=s-f\right\}\right\rangle, \\
& N(f ; k, s)=[s ! k ! /(k+2 f) !(s-f) !]^{1 / 2} \\
& V_{0}|k, s ; f\rangle=\left(l_{0}+f\right)|k, s ; f\rangle \\
& |k, s\rangle=\left|n_{1}=k, n_{0}=s\right\rangle, \quad V_{-}|k, s\rangle=0, \quad k=0,1, s \geq 0 .
\end{aligned}
$$

Evidently, Eqs. (7) explicitly manifest the $s u_{p d}(2)$ cluster structure of the Fock states $\left|n_{1}, n_{0}\right\rangle$ and specify $R_{0}$ as the "lowest" weight operator and $|k, s\rangle$ as the "lowest" weight state.

This $s u_{p d}(2)$ cluster reformulation of the model enables one to use the $s u_{p d}(2)$ formalism for getting representations of the model evolution operator $U_{H}(t)=$ $\exp (-i H t / \hbar)$ which facilitate analysis of the model dynamics and calculations of temporal dependences $\langle O(t)\rangle=\operatorname{Tr}\left[U_{H}(t) \rho U_{H}^{\dagger}(t) O\right]$ for arbitrary physical observables $O ; \rho=\rho(0)$ is the density operator of the initial quantum state $[6,11]$. One of such adequate representations has a diagonal form $[11,12]$

$$
\begin{aligned}
& U_{H}(t)=\sum_{k, s ; v} e^{-i t \epsilon(k, s ; v)}\left|E_{v}(k, s)\right\rangle\left\langle E_{v}(k, s)\right|, \\
& E_{v}(k, s)=\hbar \epsilon(k, s ; v)=\hbar\left[C\left(l_{1}\right)+\lambda_{v}(k, s)\right],
\end{aligned}
$$

where $E_{v}(k, s)$ and $\left\{\left|E_{v}(k, s)\right\rangle\right\}$ are eigenvalues and complete sets of orthonormalized eigenvectors of the Hamiltonian (5) respectively:

$$
H\left|E_{v}(k, s)\right\rangle=E_{v}(k, s)\left|E_{v}(k, s)\right\rangle, \quad\left\langle E_{v}(k, s) \mid E_{v^{\prime}}\left(k^{\prime}, s^{\prime}\right)\right\rangle=\delta_{k, k^{\prime}} \delta_{s, s^{\prime}} \delta_{v v^{\prime}} .
$$

In the basis $\left\{\left|E_{v}(k, s)\right\rangle\right\}$ quantum expectations $\langle O(t)\rangle$ are given as follows [11]:

$$
\begin{gathered}
\langle O(t)\rangle=\sum_{k, s, v ; k^{\prime}, s^{\prime} v^{\prime}}\left\langle E_{v}(k, s)|\rho| E_{v^{\prime}}\left(k^{\prime}, s^{\prime}\right)\right\rangle\left\langle E_{v^{\prime}}\left(k^{\prime}, s^{\prime}\right)|O| E_{v}(k, s)\right\rangle \times \\
\times e^{i t\left[\epsilon\left(k^{\prime}, s^{\prime} ; v^{\prime}\right)-\epsilon(k, s ; v)\right]},
\end{gathered}
$$

and, hence, the main problem is in solving the eigenproblem (9). 


\section{Lie-algebraic schemes for finding diagonal $s u_{p d}(2)$ forms of the model evolution operator: an exact algorithm and cluster quasi- classical approximations}

In the case, when the PLA structure polynomial $\psi\left(V_{0} ; R_{1}\right)$ is quadratic in $V_{0}$ and PLA $s u_{p d}(2)$ is reduced to the familiar Lie algebra $\operatorname{su}(2)=\operatorname{Span}\left\{Y_{0}, Y_{ \pm}\right.$: $\left.\left[Y_{0}, Y_{ \pm}\right]= \pm Y_{ \pm},\left[Y_{-}, Y_{+}\right]=2 Y_{0}\right\}$ the eigenvalue problem (9) is solved exactly with the help of the $S U(2)$ displacement operators $S_{Y}(\xi=r g /|g|)=$ $\exp \left(\xi Y_{+}-\xi^{*} Y_{-}\right)[21]$ in terms of simple analytical expressions [12]. However, it is not the case for the model under study in view of the absence of explicit expressions for matrix elements $\left\langle k, s ; f\left|\exp \left(\sum_{i} a_{i} V_{i}\right)\right| k, s ; f^{\prime}\right\rangle[6,15]$. Nevertheless, the $s u_{p d}(2)$ formalism enables one get convenient (for physical applications) calculation schemes, algorithms and analytical expressions for exact and approximate solutions of this problem.

A Lie-algebraic scheme for finding exact solutions of the eigenproblem (9) is based on looking for eigenfunctions $\left|E_{v}(k, s)\right\rangle$ on each subspace $L(k, s)$ in the form $[14,15]$

$$
\begin{aligned}
& \left|E_{v}(k, s)\right\rangle=\sum_{f=0}^{s} Q_{f}^{v}(k, s)|k, s ; f\rangle=\sum_{f=0}^{s} \tilde{Q}_{f}^{v}(k, s) V_{+}^{f}|k, s\rangle, \\
& \tilde{Q}_{f}^{v}(k, s)=N(f ; k, s) Q_{f}^{v}(k, s),
\end{aligned}
$$

where amplitudes $Q_{f}^{v}(k, s)$ satisfy the orthonormalization and completeness conditions:

$$
\sum_{f=0}^{s} Q_{f}^{v}(k, s) Q_{f}^{v^{\prime}}(k, s)=\delta_{v v^{\prime}}, \quad \sum_{v=0}^{s} Q_{f}^{v}(k, s) Q_{f^{\prime}}^{v}(k, s)=\delta_{f f^{\prime}} .
$$

Then, inserting Eq. (11) for $\left|E_{v}(k, s)\right\rangle$ and Eq. (5) for $H$ in Eq. (9) and using Eqs. (3), (7), one gets a set of recurrence relations at fixed $k=0,1 ; s=0,1, \ldots$

$$
\begin{aligned}
& g^{*} \psi\left(l_{0}+f+1 ; l_{1}\right) \tilde{Q}_{f+1}^{v}(k, s)= \\
& \left.\quad=\left[\lambda_{v}(k, s)-\Delta\left(f+l_{0}\right)\right] \tilde{Q}_{f}^{v}(k, s)\right)-g \tilde{Q}_{f-1}^{v}(k, s), \\
& f, v=0, \ldots, s, \\
& \psi\left(l_{0}+f+1 ; l_{1}\right)=(k+2 f+2)(k+1+2 f)(s-f) .
\end{aligned}
$$

These relations along with the boundary conditions $\tilde{Q}_{-1}^{v}(k, s)=0=\tilde{Q}_{s+1}^{v}(k, s)$ determine amplitudes $Q_{f}^{v}(k, s)$ and eigenenergies $E_{v}(k, s)$ from solutions of the Sturm-Liouville spectral problem $[6,14]$ 


$$
\begin{aligned}
& P_{f+1}(\lambda)=\left[\lambda-\Delta\left(f+l_{0}\right)\right] P_{f}(\lambda)-|g|^{2} \psi\left(l_{0}+f ; l_{1}\right) P_{f-1}(\lambda), \\
& f=0, \ldots, s ; \quad P_{0}(\lambda)=1, \\
& P_{-1}(\lambda)=0=\left[\lambda-\Delta\left(s+l_{0}\right)\right] P_{s}(\lambda)-|g|^{2} \psi\left(l_{0}+s ; l_{1}\right) P_{s-1}(\lambda),
\end{aligned}
$$

for finding non-classical orthogonal (in view of (12)) polynomials

$$
P_{f}(\lambda)=\frac{\left(g^{*}\right)^{f} \tilde{Q}_{f}(k, s ; \lambda)}{N^{2}(f ; k, s) \tilde{Q}_{0}(k, s ; \lambda)}=\frac{\left(g^{*}\right)^{f} Q_{f}(k, s ; \lambda)}{N(f ; k, s) Q_{0}(k, s ; \lambda)}
$$

of the discrete variable $\lambda$ on the non-uniform lattice $\left\{\lambda_{v}(k, s)\right\}_{v=0}^{s}[14]$. Indeed, Eqs. (14), (15),(16) provide the following algorithm for solving the eigenproblem (9).

i) Using the recursive formula (14) with the boundary values from Eq. (15) one calculates the polynomial sequence $\left\{P_{f}(\lambda)\right\}_{f=0}^{s}$.

ii) Inserting $P_{s-1}(\lambda), P_{s}(\lambda)$ in the last equality in (15) one gets the algebraic equation with respect to $\lambda$; its solution yields the sequence $\left\{\lambda_{v}(k, s) \equiv \lambda_{v}\right\}_{v=0}^{s}$ of admissible values of the spectral parameter $\lambda$ and the appropriate energy spectrum $\left\{E_{v} \equiv E_{v}(k, s)\right\}_{v=0}^{s}$.

iii) For each value $\lambda_{v}(k, s)$ using $\left\{P_{f}(\lambda)\right\}_{f=0}^{s}$ and Eq. (16) one finds the sequence $\left\{Q_{f}^{v}(k, s) \equiv Q_{f}\left(k, s ; \lambda_{v}\right)\right\}_{f=1}^{s}$ of all amplitudes as functions of the only undetermined quantity $Q_{0}\left(\lambda_{v}\right) \equiv Q_{0}\left(k, s ; \lambda_{v}\right)$ which, in turn, is found from the normalization condition of Eqs. (12).

This algorithm has been realized in [22] with the help of the REDUCE procedure SOLVE for $s \leq 100$ and the conventional FORTRAN subroutines of EISPARK package (with applying the multiprecision package [23]) for $s \geq 100$.

The routine package developed enables us to implement numerical calculations of model dynamics for $s \leq 160$ but it is unsuitable for practical calculations with larger $s$ in view of multiprecision computer limitations. Therefore in [11] an approximate analytical solution of the problem (9) has been suggested. It is given by the $S U(2)$-quasiclassical eigenfunctions

$$
\begin{aligned}
& \left|E_{v}^{q c}(k, s ; \xi=r g /|g|)\right\rangle=\exp \left(\xi Y_{+}-\xi^{*} Y_{-}\right)|k, s ; v\rangle=\sum_{f} S_{f v}^{j}(\xi)|k, s ; f\rangle,(17 \\
& Y_{0}=V_{0}-l_{0}-j, \quad Y_{+}=V_{+}\left[2\left(s+2 Y_{0}+2 k+1\right)\right]^{-\frac{1}{2}}=\left(Y_{-}\right)^{+}, 2 j=s \\
& Y_{ \pm, 0} \in \operatorname{su}(2), \quad S_{f v}^{j}(\xi) \equiv Q_{f}^{v ; a p}(k, s)=\left(\frac{g}{|g|}\right)^{f-v} d_{-j+f,-j+v}^{j}(2 r),
\end{aligned}
$$

and eigenenergies

$$
\begin{aligned}
& E_{v}^{q c}(k, s ; \xi) \equiv\left\langle E_{v}^{q c}(k, s ; \xi)|H| E_{v}^{q c}(k, s ; \xi)\right\rangle=\hbar\left[C\left(l_{1}\right)+\lambda_{v}^{q c}(k, s ; r)\right] \\
& \lambda_{v}^{q c}(k, s ; r)=\Delta\left[j+l_{0}-(j-v) \cos 2 r\right]+
\end{aligned}
$$




$$
\begin{aligned}
& +2|g| \sum_{f=0}^{s} \sqrt{(s-f)(f+1) 2(2 k+1+2 f)} d_{-j+f,-j+v}^{j}(2 r) d_{-j+f+1,-j+v}^{j}(2 r) \\
& \approx \Delta\left[j+l_{0}-(j-v) \cos 2 r\right]- \\
& -2|g|(j-v) \sin 2 r \sqrt{2[s+2 k+1+(-s+2 v) \cos 2 r]}=\lambda_{v}^{c m f}(k, s ; r),(19)
\end{aligned}
$$

where $d_{m, n}^{j}(2 r)$ are the $S U(2) d$ - functions expressed in terms of the Gauss hypergeometric function ${ }_{2} F_{1}[11]$. Approximate values $\lambda_{v}^{c m f}(k, s ; r)$ in (19) are calculated in the cluster mean-field approximation: $\left\langle k, s ; f\left|F\left(Y_{\alpha}\right)\right|[k, s ; f\rangle=\right.$ $F\left(\left\langle k, s ; f\left|Y_{\alpha}\right| k, s ; f\right\rangle\right)$, and values of the parameter $r$ in (17)-(19) are found from energy-stationarity-conditions and/or from minimizing a proximity measure between exact Hamiltonian $H$ and its $S U(2)$ - quasiclassical approximation

$$
H^{q c}(\xi)=\sum_{v, k, s} E_{v}^{q c}(k, s ; \xi)\left|E_{v}^{q c}(k, s ; \xi)\right\rangle\left\langle E_{v}^{q c}(k, s ; \xi)\right|
$$

A standard measure for such estimates on the subspaces $L(k, s)$ is defined with the help of the unitarily invariant euclidean operator norm [24] as follows [11]

$$
\delta_{H}^{2}(k, s)=\frac{\operatorname{Tr}_{(k, s)}\left(H-H^{q c}(\xi)\right)^{2}}{\operatorname{Tr}_{(k, s)}\left(H-C\left(l_{1}\right)\right)^{2}}=\frac{\sum_{v}\left[\left(\lambda_{v}(k, s)\right)^{2}-\left(\lambda_{v}^{q c}(k, s ; r)\right)^{2}\right]}{\sum_{v}\left(\lambda_{v}(k, s)\right)^{2}} .
$$

This approximation has been used in [11] for calculating approximate expressions $\left\langle Y_{0}(t)\right\rangle^{q c}$ of the temporal dependences $\left\langle Y_{0}(t)\right\rangle$ determining, in accordance with Eqs. (2), (18), the dynamics of the field-mode populations:

$$
\left\langle N_{0}(t)\right\rangle=\frac{\bar{s}}{2}-\left\langle Y_{0}(t)\right\rangle, \quad\left\langle N_{1}(t)\right\rangle=\bar{s}+\bar{k}+2\left\langle Y_{0}(t)\right\rangle
$$

for different types of initial states; here $\bar{s}=\operatorname{Tr}\left[\rho\left(R_{1}-R_{0}\right)\right], \bar{k}=\operatorname{Tr}[\rho K]$ and the quantity $\left\langle Y_{0}(t)\right\rangle$ is calculated with the help of Eq. (10) for $O=$ $Y_{0}$. Specifically, in the case of the $S U(2)$-quasiclassical cluster initial state |\rangle$_{C}$ of the form (17), (18) with $\xi=0, v=0$, belonging to a fixed "optical atom" $L(k, s)$ with $\left\langle N_{0}(0)\right\rangle=s,\left\langle N_{1}(0)\right\rangle=k$, Eq. (10) yields an approximate analytical expression [11]

$$
\left\langle Y_{0}(t)\right\rangle_{C}^{q c} \approx-\frac{1}{2}\left\{1+(s-1) A_{k, s}(t) \cos \left[\Omega_{L}(k, s) t-(s-1) \Phi_{k, s}(t)\right]\right\}
$$

with

$$
\Omega_{L}(k, s)=4|g| \sqrt{\left(1-\frac{1}{s}\right)\left(\frac{s}{2}+k+\frac{1}{2}\right)}, \quad \Omega_{l}(k, s)=\frac{4|g| \sqrt{s-1}}{s \sqrt{2 s+4 k+2}}
$$




$$
A_{k, s}(t)=\left[\cos ^{2} \Omega_{l}(k, s) t+\frac{\sin ^{2} \Omega_{l}(k, s) t}{s}\right]^{\frac{s-1}{2}}, \quad \tan \Phi_{k, s}(t)=\frac{\tan \Omega_{l}(k, s) t}{\sqrt{s}}
$$

which exhibits a high-frequency $\left(\Omega_{L}(k, s)\right)$ periodic dynamics with a slow $\left(\Omega_{l}(k, s)\right)$ periodic modulation in the phase and amplitude, i.e. an occurrence of a specific temporal coherent structure (described in terms of elliptic functions too) [11,12]. At the same time for general initial states $\|\rangle \in L(H)$ and having non-zero projections on all subspaces $L(k, s)$, e.g., for Glauber coherent states [11], analogous calculations lead to series containing weighted sums of terms like those given by Eqs. $(23),(24)$ that corresponds to occurrences of coherence-decoherence phenomena like "collapse-revivals" revealed in $[18,20]$ by means of other methods.

However, according to the general quasiclassicality theory [25] all approximations (17)-(19) (and, hence, (23), (24)) are valid only for large values of $s$, and, besides, the measure (21) gives only a global rather than local characteristic of the approximate energy spectra $\left\{E_{v}^{q c}(k, s ; \xi)\right\}$ that does not allow to feel their important symmetry properties and local peculiarities related to "energy errors"

$$
\Delta E_{v}(k, s)=\hbar\left[\lambda_{v}(k, s)-\lambda_{v}^{q c}(k, s ; r)\right] \equiv \delta E_{v}(k, s) \cdot E_{v}(k, s) .
$$

Therefore, we implemented numerical comparisons of both exact and approximate results in order to estimate the applicability range of the quasiclassical approximation (17)-(19).

\section{Comparison of exact and approximate calculations in the reso- nance case}

In order to examine the efficiency of calculation schemes and the algorithm given above we tested them by means of computer experiments for the resonance case determined by $H$ from Eq. (11) with $\omega_{0}=2 \omega_{1}, C\left(l_{1}\right)=\omega_{1}(k+$ $2 s), \Delta=0$.

First of all we calculated exact values $\lambda_{f}(k, s), Q_{f}^{v}(k, s)$ according to the algorithm of Section 3 and their approximations $\lambda_{v}^{c m f}\left(k, s ; r_{i}\right), S_{f v}^{j}(\xi)$ according to Eqs. (17)-(19) for $g=1, k=0,1, s=20,10^{2}, 5 \cdot 10^{2}, 10^{3}, 10^{4}$. Values of the fitting parameter $r$ were determined from energy-stationarity-conditions: $2 r_{1}=$ $\arccos \frac{1}{3}$ (optimizing only the upper part of spectra) [11], $2 r_{3}=\arccos 0=\frac{\pi}{2}$ (quasi-linear approximation) [14] and from minimizing the proximity measure (21): $2 r_{2}=\arccos \frac{1}{\sqrt{s}}$ ("smooth" cluster mean-field approximation) [11]; herewith $\lambda_{v}^{c m f}\left(k, s ; \mp r_{1}\right)$ means that we take $r=-r_{1}$ in the first half of spectra 
and $r=r_{1}$ in the second one. To estimate the accuracy of approximations we also used non-invariant measures

$$
\begin{aligned}
& \delta_{E}^{2}(k, s)=\frac{\sum_{v=0}^{s}\left[\left(\lambda_{v}(k, s)-\lambda_{v}^{c m f}(k, s ; r)\right]^{2}\right.}{\sum_{v=0}^{s}\left(\lambda_{v}(k, s)\right)^{2}}, \\
& \delta_{E_{u p}}^{2}(k, s)=\frac{\sum_{v=s / 2}^{s}\left[\left(\lambda_{v}(k, s)-\lambda_{v}^{c m f}(k, s ; r)\right]^{2}\right.}{\sum_{v=s / 2}^{s}\left(\lambda_{v}(k, s)\right)^{2}},
\end{aligned}
$$

to characterize more precisely (in comparison with Eq. (21)) energy spectra and standard (related to the Fubini-Study metric [26] in $L(k, s)$ ) measures

$$
\cos (\mathbf{S}, \mathbf{Q})_{\left[l_{i}\right] ; v} \equiv \sum_{f} S_{f v}^{j} Q_{f}^{v}(k, s), \quad \delta_{e f}^{2}(k, s ; v)=1-\left|\cos (\mathbf{S}, \mathbf{Q})_{k, s ; v}\right|^{2}(27)
$$

(or associated graphic representations via "overlap areas") to estimate an "approximation quality" for eigenfunctions. Some of typical results of these numerical calculations are presented in Table 1 and Figs.1,2.

Table 1. Multiplets $\left\{\lambda_{v}(k=0, s)\right\}_{v=0}^{s},\left\{\lambda_{v}^{c m f}\left(0, s ; r_{i}\right)\right\}_{v=0}^{s}$ with the level step $\Delta v=10$ for $s=10^{2}$.

$\begin{array}{llllll}v & \lambda_{v}(0, s) & \lambda_{v}^{c m f}\left(0, s ; r_{1}\right) & \lambda_{v}^{c m f}\left(0, s ; \mp r_{1}\right) & \lambda_{v}^{c m f}\left(0, s ; r_{2}\right) & \lambda_{v}^{c m f}\left(0, s ; r_{3}\right) \\ 0 & -1536.9 & -1096.7 & -1545.3 & -1482.4 & -1421.2 \\ 10 & -1151.7 & -919.6 & -1205.2 & -1175.2 & -1137.0 \\ 20 & -798.1 & -720.0 & -880.0 & -873.3 & -852.7 \\ 30 & -480.3 & -499.3 & -570.2 & -576.7 & -568.5 \\ 40 & -205.5 & -259.0 & -276.7 & -285.6 & -284.2 \\ 50 & 0.0 & 0.0 & 0.0 & 0.0 & 0.0 \\ 60 & 205.5 & 276.7 & 276.7 & 280.0 & 284.2 \\ 70 & 480.3 & 570.2 & 570.2 & 554.3 & 568.5 \\ 80 & 798.1 & 880.0 & 880.0 & 822.8 & 852.7 \\ 90 & 1151.7 & 1205.2 & 1205.2 & 1085.5 & 1137.0 \\ 100 & 1536.9 & 1545.3 & 1545.3 & 1342.3 & 1421.2 \\ \delta_{H}^{2}= & 10.222 & -12.220 & 0.010 & -1.000 \\ \delta_{E}^{2}= & 2.563 & 0.670 & 0.806 & 0.657 \\ \delta_{E_{\text {up }}}^{2}= & 0.670 & 0.670 & 0.944 & 0.657\end{array}$

As is seen from data given in Table 1 and Fig. 1 we have an acceptable consent of exact eigenenergies and their approximations (19) at $s \gg 1$ almost everywhere for $r=r_{i=2,3}$ and $r=\mp r_{1}$. Discrepancies between exact and approximate results in the middle parts of spectra, probably, are due to the 

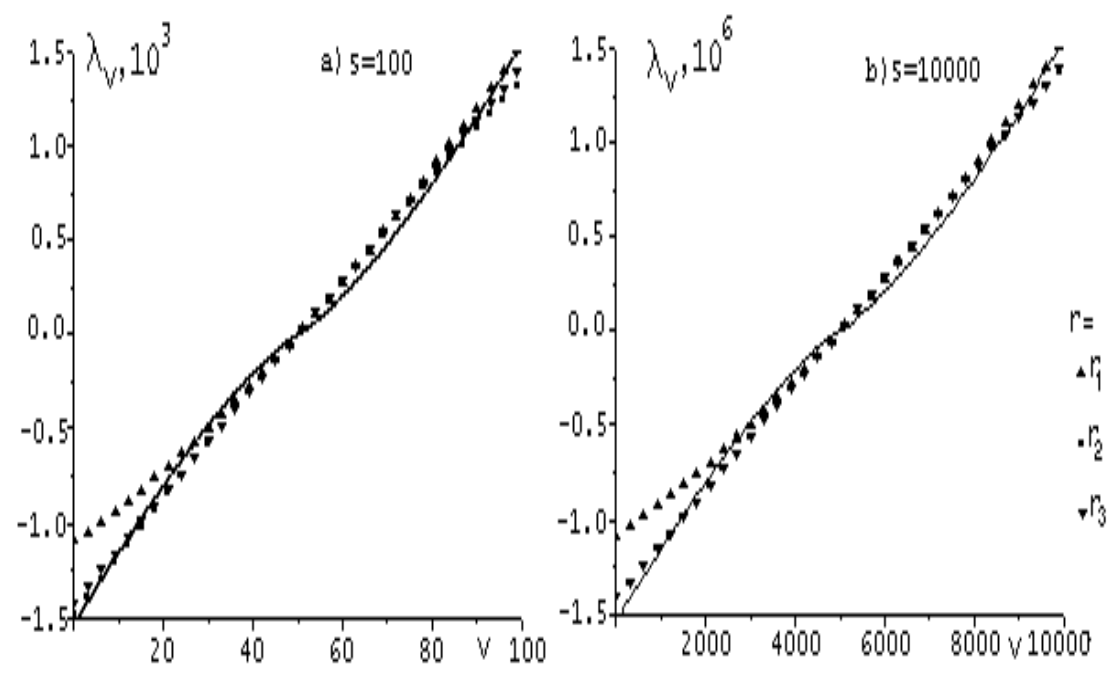

Fig. 1. Energy levels $\left.\lambda_{v}(k=0, s)\right\}$ (solid line) and their approximations (19) with $r=r_{i=1,2,3}$ plotted against the energy label $v$ for $\left.s=10^{2}(\mathrm{a})\right)$ and $\left.s=10^{4}(\mathrm{~b})\right)$.

availability of the square-root singularities in the model Hamiltonian (5) rewritten (with the help of Eqs. (18)) in terms of $Y_{\alpha}$ that is, actually, ignored in the "smooth" the su(2) - quasiclassical approximation (17)-(19) [12]. (Note also that some negative values of $\delta_{H}^{2}$ are due to using $\lambda_{v}^{c m f}$ instead of $\lambda_{v}^{q c}$ in Eq. (21) and because of calculation errors). However, the approximation with $r=\mp r_{1}$ breaks the orthogonality of eigenfunctions belonging to opposite ends of spectra whereas the quasi-linear approximation with $r=r_{3}$ leads to equidistant spectra within fixed subspaces $L(k, s)$. Therefore, in spite of the spectrum symmetry breaking, the most satisfactory quasiclassical approximation is given by Eqs. (17)-(19) with $r=r_{2}$ that minimizes $\delta_{H}^{2}$. Note that the spectrum asymmetry at $r=r_{2}$ and related shifts between amplitude values $Q_{f}^{v}(k, s)$ and $S_{f v}^{j}(\xi)$ (see Fig. 2) are due to using "smooth" $s u(2)$ - quasiclassical eigenfunctions (17) [12].

Besides the verifications above we also performed calculations of temporal dependences of the quantity $\left\langle Y_{0}(t)\right\rangle_{C}$ and related dynamics of the normalized average photon numbers $\left\langle N_{i}(t)\right\rangle_{C} / s$. Herewith exact dependencies were calculated with the help of routine package above, whereas approximate calculations were implemented using approximate expressions (17), (18), (19) for eigenvalues and eigenfunctions. Results of such calculations for $s=10^{2}, k=0$ against the dimensionless time $\tau=g t \sqrt{2 s}$ are plotted in Fig. 3 where we compare exact results with the quasiclassical approximations obtained with the help of Eqs. (17)-(19) with $r=r_{i=1,2}$ and (23)-(24). 

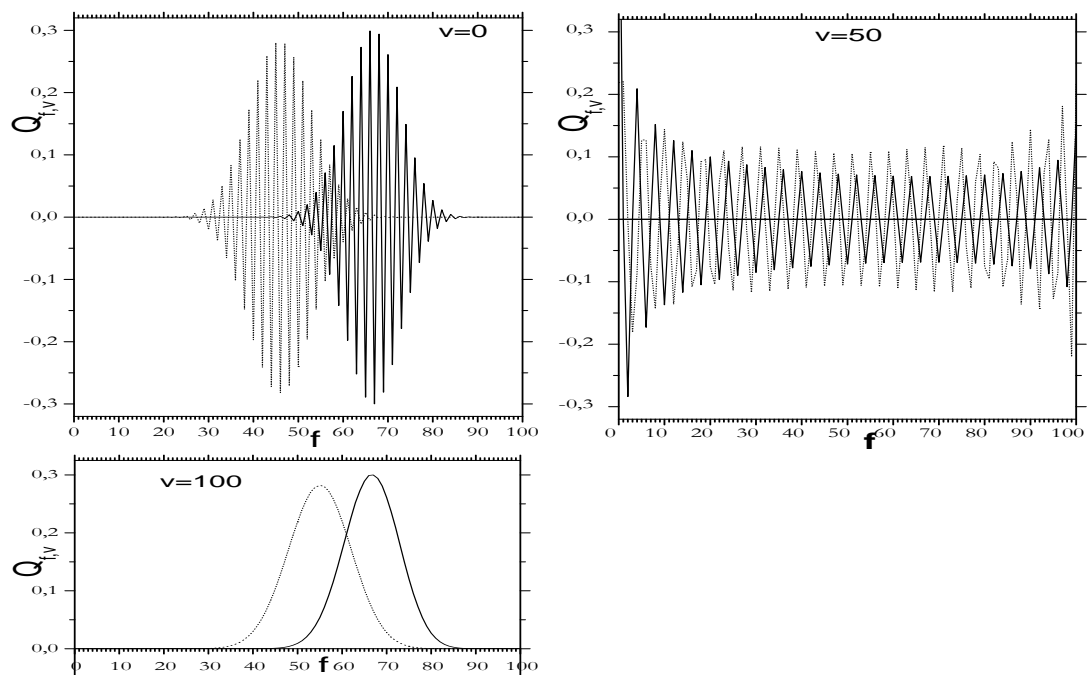

Fig. 2. Amplitudes $Q_{f}^{v}(k, s)$ as functions in $f=0, \ldots, s$ at $k=0, v=0,50,100$ (solid lines) and their quasiclassical approximations $S_{v f}^{j}\left(r_{2} g /|g|\right)$ (dashed lines).

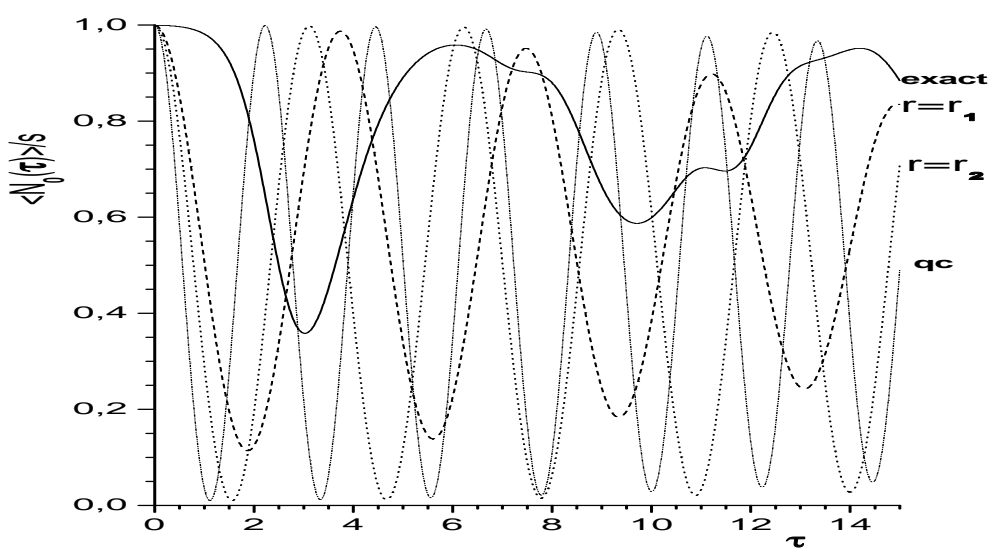

Fig. 3. Normalized average photon number $\left\langle N_{0}(t)\right\rangle_{C} / s$ in the fundamental mode plotted against time $\tau$ for $k=0, s=10^{2}$ : (exact) is results of (22) and their approximations (qc) and $\left(r=r_{i=1,2}\right)$ calculated by (23) and (17)-(19) with $r=r_{i=1,2}$.

Evidently, the graphic representations of Fig.3 enable us to reveal transparently a double-periodic component in the exact multi-frequency dynamics of $\left\langle Y_{0}(t)\right\rangle$ and $\left\langle N_{0}(t)\right\rangle$ that is rather well described by Eqs. (23), (24) or (17)-(19) at $r=r_{2}$ (and at $r=r_{1}$ ). Note that an availability of this important dynamic feature is displayed clearer when the characteristic parameter $s$ increases (in accordance with the general quasiclassical theory [25]). 


\section{Conclusion}

So, our numerical calculations given in Section 4 show a rather good qualitative consent of exact and approximate results at $s \gg 1$ and at relevant choices of the fitting parameter $r$ in (17)-(19). However, partial quantitative discrepancies of them require further improvements of the quasiclassical approximations used. In particular, the approximate solutions of the eigenproblem (9) can be improved by means of: 1) using less smooth (in comparison with (17), (18)) generalized coherent states of the $s u_{p d}(2)$ algebra as quasiclassical eigenfunctions (cf. [12]) and 2) exploiting the standard [24] or special (e.g., developed in $[27,28])$ algebraic perturbative and iterative algorithms or modifications of the algebraic "dressing" schemes [6]. Then these improvements (along with the exact calculation schemes developed above) can be used for a more detail analysis (like those implemented in $[7-9,16-20]$ ) of the model under consideration in all ranges of the parameter $s$ and for arbitrary initial states. It is also of interest to compare results obtained (and their improvements) with those of

[11] based on an alternative $S U(2)$ form of $U_{H}((t)$ as well as with calculations performed in [29] using the formalism of the $q$ - deformed Lie algebra $s u_{q}(2)$. The work along these lines is in progress.

\section{References}

[1] J.A. Armstrong, N. Bloembergen, J. Ducuing, and P.S. Pershan, Phys. Rev., 1271918 (1962); N. Bloembergen, Nonlinear Optics, W. A. Benjamin, New York (1965).

[2] J.H. Eberly, N.B. Narozhny, J.J. Sanchez-Mondragon, Phys. Rev. Lett., 44, 1329 (1980).

[3] Y.R. Shen, The principles of nonlinear optics (Wiley, New York, 1984)

[4] J. Perina, Quantum Statistics of Linear and Nonlinear Optical Phenomena. (Reidel, Dordrecht 1984).

[5] V.P. Karassiov, L.A. Shelepin, Trudy FIAN [P.N. Lebedev Inst. Proc], 144, 124 (1984); V.P. Karassiov, J. Sov. Laser Res., 12, 147 (1991).

[6] V.P. Karassiov and A.B. Klimov, Phys. Lett., A 189, 43 (1994).

[7] Z.Y. Ou, Phys. Rev., A 492106 (1994).

[8] R.D. Li, P. Kumar, Phys. Rev., A 492157 (1994).

[9] S.M. Chumakov and Kozierowski, Quantum Semiclass. Opt. 8, 775 (1996); A. Bandilla, G. Drobny and I. Jex, Phys. Rev., A 53507 (1996).

[10] N. Debergh, J. Phys., A 30, 5239 (1997); A 31, 4013 (1998). 
[11] V.P. Karassiov, Phys. Lett., A 238, 19 (1998); J. Rus. Laser Res., 20, 239 (1999).

[12] V.P. Karassiov, J. Rus. Laser Res., 21, 370 (2000); Phys. Atom. Nucl., 63, 648 (2000); Optika i Spektr., 91, 543 (2001).

[13] A.B. Klimov, L.L. Sanchez-Soto, Phys. Rev., A 61, 063802 (2000).

[14] V.P. Karassiov, Teor. Mat. Fiz., 95, 3 (1993); J. Phys., A 27, 153 (1994).

[15] V.P. Karassiov, Rep. Math. Phys., 40,235 (1997); Czech. J. Phys.,48, 1381 (1998).

[16] M. Kozierowski, R. Tanas, Opt. Commun. 21229 (1977)

[17] L. Mandel, Opt. Commun. 42437 (1982).

[18] I.Sh. Averbukh, N.F. Perel'man, Sov. Phys.- JETP 96818 (1989); Phys. Lett., A 139, 449 (1989).

[19] S.P. Nikitin, A.V. Masalov, Quantum Opt. 3105 (1991).

[20] M.K. Olsen, et al., Phys. Rev., A 61021803 (2000).

[21] A.M. Perelomov, Generalized Coherent States and Their Applications. (Nauka, Moscow, 1987).

[22] V.P. Karassiov, A.A. Gusev, S.I. Vinitsky, E-archive: quant-ph/ 0105152 (2001); in: Proc. XXIII Inter. Coll. Group Theor. Meth. Phys. ICGTM-23 (Dubna, July 28-August 5, 2000). (JINR, Dubna, in press).

[23] D.H. Bailey, ACM Trans. Math. Softw. 19, 288 (1993); 21, 379 (1995).

[24] T. Kato, Perturbation Theory for Linear Operators. (Springer, Berlin e.a., 1965); P. Lankaster, Theory of Matrices. (Academic, New York-London, 1969).

[25] L.G. Jaffe, Rev. Mod. Phys., 54407 (1982); A. Chatterjee, Phys. Rep., 186, 249 (1990).

[26] D.N. Page, Phys. Rev., A 363479 (1987); S. Kobayashi and K. Nomizu, Differential Geometry, vol. 2 (Interscience, New York,1969).

[27] A. Gusev, V. Samoilov, V. Rostovtsev and S. Vinitsky, in: Computing Algebra in Scientific Computing: Proc. Workshop CASC 2000, V.G. Ganzha, E.W. Mayr, E.V. Vorozhtsov (Eds.) (Springer, Berlin e.a., 2000), p. 219.

[28] E.A. Grebenikov, Yu. A. Mitropolsky, Yu. A. Ryabov, Introduction into the Resonance Analytic Dynamics. (Janus-K, Moscow,1999).

[29] A. Ballesteros and S.M. Chumakov, J. Phys., A32 6261 (1999). 